\title{
3D-Stereoscopic Immersive Analytics Projects at Monash University and University of Konstanz
}

\author{
Björn Sommer ${ }^{1,2^{*}}$, David G. Barnes ${ }^{3,4}$, Sarah Boyd ${ }^{3}$, Thomas Chandler ${ }^{2}$, Maxime Cordeil' ${ }^{2}$, Tobias Czauderna ${ }^{4}$, Mathias Klapperstück ${ }^{4}$, \\ Karsten Klein ${ }^{1,2}$, Toan D. Nguyen ${ }^{4}$, Hieu Nim ${ }^{3,5}$, Kingsley Stephens ${ }^{2,3}$, Dany Vohl ${ }^{6}$, Stephen Wang ${ }^{7}$, Elliott Wilson $^{2}$, Yan Zhu $^{8}$, Jian Lia $^{8}$, \\ Jon McCormack ${ }^{2}$, Kim Mariott ${ }^{2}$, Falk Schreiber ${ }^{1,3}$ \\ ${ }^{1}$ Department of Computer and Information Science, University of Konstanz, Germany, 78457 Konstanz, Germany \\ ${ }^{2}$ Faculty of Information Technology, Monash University, 3145 Caulfield, Australia \\ ${ }^{3}$ Faculty of Information Technology, Monash University, 3800 Clayton, Australia \\ ${ }^{4}$ Monash Immersive Visualization Platform, Monash University, 3800 Clayton, Australia \\ ${ }^{5}$ Australian Regenerative Medicine Institute, Monash University, 3800 Clayton, Australia \\ ${ }^{6}$ Centre for Astrophysics \& Supercomputing, Swinburne University of Technology, 3122 Hawthorn, Australia \\ ${ }^{7}$ International Tangible Interaction Design Lab \& Department of Design, Monash University, 3145 Caulfield, Australia \\ ${ }^{8}$ Department of Microbiology, Monash University, 3800 Clayton, Australia \\ "Corresponding author: bjoern@CELLmicrocosmos.org
}

\begin{abstract}
Immersive Analytics investigates how novel interaction and display technologies may support analytical reasoning and decision making. The Immersive Analytics initiative of Monash University started early 2014. Over the last few years, a number of projects have been developed or extended in this context to meet the requirements of semi- or full-immersive stereoscopic environments. Different technologies are used for this purpose: CAVE2 ${ }^{\mathrm{TM}}$ (a 330 degree large-scale visualization environment which can be used for educative and scientific group presentations, analyses and discussions), stereoscopic Powerwalls (miniCAVEs, representing a segment of the CAVE2 and used for development and communication), Fishtanks, and/or HMDs (such as Oculus, VIVE, and mobile HMD approaches). Apart from CAVE2 ${ }^{\mathrm{TM}}$ all systems are or will be employed on both the Monash University and the University of Konstanz side, especially to investigate collaborative Immersive Analytics. In addition, sensiLab extends most of the previous approaches by involving all senses, $3 D$ visualization is combined with multi-sensory feedback, $3 D$ printing, robotics in a scientific-artistic-creative environment.
\end{abstract}

\section{Introduction}

Immersive Analytics is an emerging field which investigates how novel interaction and display technologies may support analytical reasoning and decision making [1]. Whereas Visual Analytics [2], [3] usually tend to provide 2D visualization-based solutions, Immersive Analytics makes broad use of 3D visualization and therefore largely profits from Stereoscopic $3 D$ $(S 3 D)$ technologies [4]. And this is usually done by immersing the user into virtual environments representing the data to be analyzed. Moreover, collaboration support as well as multi-sensory feedback play important roles in this context.

We show here how recent technologies are applied to a number of scientific projects which are all being developed at Monash University and/or the University of Konstanz. First, we will present the involved visualization facilities. In the preceding chapter, the applied S3D visualization technologies are introduced. Then, a selection of established and/or promising projects will be discussed which are being developed by the aforementioned visualization facilities. Finally, the paper will be concluded with a short outlook.

\section{Visualization Facilities}

First, the different visualization facilities will be introduced. The boundaries are floating between the Immersive Analytics Initiative, the sensiLab, as well as the Monash Immersive Visualization Platform. And as collaboration is an important aspect of Immersive Analytics, this is an optimal environment to conduct Immersive Analytics research.

\section{Immersive Analytics Initiative}

The Immersive Analytics Initiative of Monash University and the University of Konstanz represents an open Australian/German collaboration between researchers from various fields. It was established in Melbourne a short time ago and since 2016 we started to establish it also in Konstanz.

The Immersive Analytics initiative at Monash University is making use of the Monash Immersive Visualization Platform, as well as of the broad opportunities provided by the sensiLab. In Konstanz, we started to create our immersive research lab based on approaches we developed in Melbourne. Now, we are collaborating on various research topics, continuing and extending our started projects. Immersive analytics in the context of our joint initiative between Monash University and Konstanz University is being trialed in five application areas:

- Life Sciences (University of Konstanz/Monash University),

- Disaster Management (Monash University),

- Business Intelligence \& Logistics (Monash University),

- Design \& Fabrication (Monash University), and

- Education (Monash University/University of Konstanz).

\section{Monash Immersive Visualization Platform}

The Monash Immersive Visualization Platform (MIVP) operates the CAVE2 ${ }^{\mathrm{TM}}$ at the New Horizons building in Clayton/Melbourne. The CAVE2 provides one of the world's most advanced immersive hybrid $2 \mathrm{D}$ and $3 \mathrm{D}$ virtual reality environments and will be introduced in the following chapter.

MIVP is supported by a team of programmers which are experts in data and geometry transfer for visualization purposes and collaborate with researchers and industry to understand their specific requirements. Conventional visualizations as well as Immersive Analytics-requiring approaches are covered by MIVP. 


\section{sensilab}

Immersive analytics research at Monash is underpinned by the sensiLab at Caulfield/Melbourne. sensiLab provides an environment in which immersive analytics researchers can quickly prototype ideas, develop new interactive devices and explore lower cost alternatives to the previously-mentioned CAVE2 with the aim of "democratizing" immersive experiences. Mobile devices making use of google cardboard, S3D TVs, as well as HMDs are used in this laboratory.

Additionally, 3D modeling, 3D printing, electronics, robotics, spatialized sound and haptic feedback play an important role in the lab's research. Whereas the other two initiatives focus more on scientific applications, sensiLab has a broader target audience. Besides researchers and university students, artists, designers, and school students can freely experiment with the latest technologies and rapidly prototype new ideas. The lab is designed to encourage creativity and play, engaging all the senses in an active, social environment that supports spontaneity and speculative design. Therefore, sensiLab represents a great place for communicating science to a broader public.

\section{Visualization Technologies}

Immersive analytics builds on technologies such as CAVEs, Powerwalls, Fishtanks or HMDs which are combined with new tracking and gesture recognition devices. In the context of our Immersive Analytics-related projects, a number of S3D visualization technologies are used. Here we would like to provide an overview of these technologies:

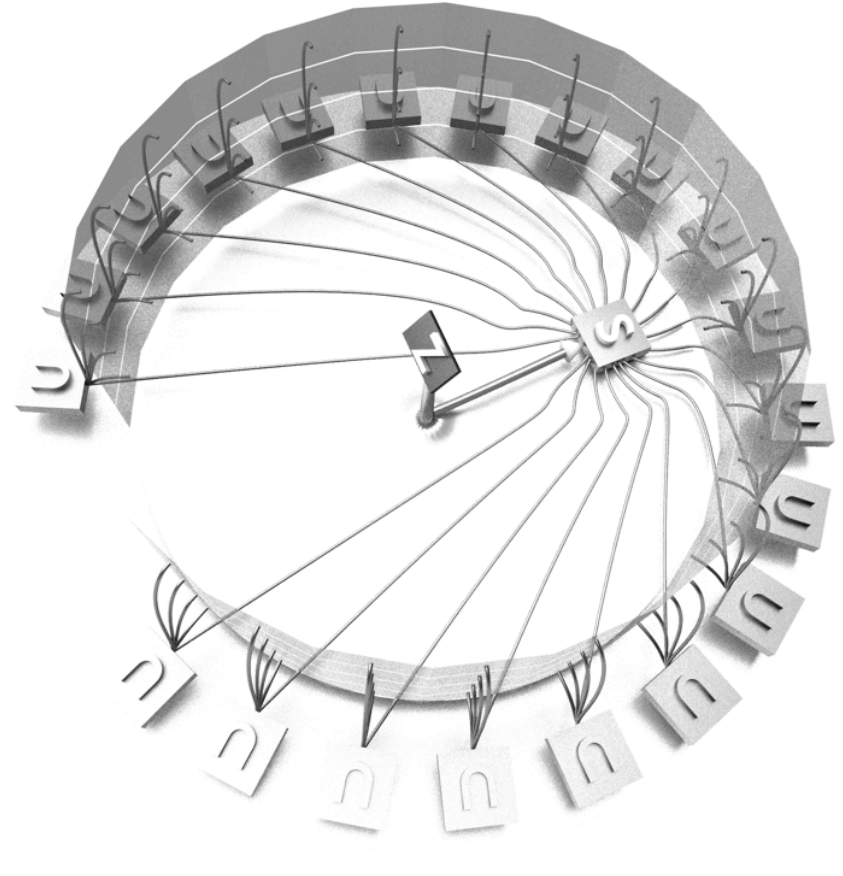

Figure 1.CAVE2: The illustration shows the 3D monitors (here: semitransparent) of the CAVE2. Four of them are each connected to a single node (n). The nodes are connected to a single head node server (s). The Stereoscopic Space Map extends the CAVE2 by the zSpace system (z) which communicates with the server.

\section{CAVES}

CAVEs (CAVE Automatic Virtual Environments) went through a different developments since this term was coined by Cruz-Neira et al. in the 1990s [5]. Whereas traditional CAVEs usually consist of at least three large stereoscopic displays placed in 90 degree angles to each other, new approaches such as the $\mathrm{CAVE} 2^{\mathrm{TM}}$ at Monash University consist of $8046^{\prime \prime}$ S3D LCDs, providing a $330^{\circ}$ panorama view [6]-[8].

In contrast to the relatively small CAVEs, CAVE2 can be used by larger audiences with approx. 20 members. Figure 1 shows the composition of the CAVE2: A central server (s) is connected to 20 nodes (n) which are each driving the graphics for four LCD monitors. A software which is supporting this configuration including S3D visualization and server-client communication is Omegalib [9]. Moreover, we will present here a new project supporting advanced volume rendering capabilities: encube.

The CAVE2 is used for a large number of different projects, for the presentation and discussion of scientific data, Human Computer Interaction (HCI) research, as well as for educational purposes.

\section{Powerwalls}

Many professional visualization laboratories provide large S3D screen setups, so-called Powerwalls. As large display screen setups, such as CAVE2, demand large investments in terms of financial, human and time resources, smaller screens setups provide a valuable alternative. A Powerwall might be a single large S3D display or a tiled display, consisting of multiple monitors. Our miniCAVE is an approach which is based on the CAVE2 technology by using, e.g., the aforementioned Omegalib in conjunction with smaller setups, such as a combination of three large S3D screens in a line (Monash University), or six S3D screens in a $2 \times 3$ configuration (Konstanz University, Figure 8 ). Referring to Figure 1, each of these configuration is driven by one server (s) connecting to three nodes $(n)$. As the development is done using Omegalib, the effort of porting projects from miniCAVE2 to CAVE2 is relatively small.

\section{Fishtanks}

Fishtanks are S3D screens featuring standard computer monitor sizes. They have a long tradition: commercial versions once started with CRT monitors in combination with shutter glasses, and then were making use of LCD technology. One which was very famous over approx. one decade was the Planar Mirror Technology ${ }^{\circledR}$ which was discontinuity a short time ago [10]. However, alternatives are available, such as more3D moreSonic ${ }^{3}$ [11], [12]. The big advantage is here that two screens projecting polarized images from two monitors are combined by using a mirror - the result is a very stable flicker-free image.

Another very good alternative is nowadays provided by $\mathrm{ZSpace}^{\mathrm{TM}}$. The $\mathrm{zSpace}$ 200, e.g., provides a passive Full HD 23" $3 \mathrm{D}$ screen in combination with an infrared-light-based head tracking system and a stylus pen for 3D interaction [13].

By using our Space Map approach (Figure 4), the zSpace can be used as a navigation device for CAVE2. As both, CAVE2 as well as the zSpace use circularly polarized glasses, their parallel use is possible. In Figure 1 is also shown that the zSpace system $z$ directly communicates with the server.

Another large advantage of the zSpace is the fact that it is - in combination with a laptop providing professional graphics - a very mobile device for display-based S3D presentations. 


\section{Head-Mounted Displays}

Head-Mounted Displays (HMDs) went through many developmental stages since their invention by Sutherland 1968 [14]. The biggest advancement occurred in 2013 when the Oculus company presented the DK1 version. Since then, the VR hype picked up and many industrial actors from the mobile (HTC) to the gaming (Sony) industry developed high-end products with high resolution and low-latency head tracking, making them usable by a vast majority of users. With the arrival of the commercial version of the Oculus Rift ${ }^{\circledR}$ and the HTC VIVE $®$ in the stores, as well as mobile versions like the Samsung Gear VR $\AA$, many users have access now to Virtual Reality technology at relative low cost. Mixed-reality devices are also developing fast. Microsoft is the first company to release in 2016 a self-contained head-set that allows holography, the MS Hololens. The Hololens embeds kinectlike technology to map the 3D environment of the user with high accuracy and displays 3D stereo images on a transparent google. With this technology, users view holograms in their environment. This type of technology is even more usable than VR headsets as users are still able to perceive their real environment, provoking less to no motion sickness and providing a rich mixed-reality experience.

Therefore, whereas CAVE-related technologies are still predominant in terms visual acuity and binocular resolution [15], the high mobility and affordability will support the success of HMDs. This technology is used by a large number of different projects which will be discussed in the following chapters.

\section{Projects}

A number of projects will be discussed which were developed at Monash University and University of Konstanz.

\section{Simulating 24 hours at Angkor Wat}

Constructed in the 12th century, Angkor Wat is a world famous heritage site and the largest religious monument on earth. In 2013, archaeological surveys [16] confirmed a grid pattern of roads and household ponds beneath the forests within the temple confines [17]. This project explores the use of an immersive virtual environment to visualize how the Angkor Wat complex might have operated almost a millennium ago. In contrast to broad scale 'virtual archaeology' studies [18] that plot changes in a site over decades or centuries, our simulation focuses on just 24 hours: a day in the medieval life of Angkor Wat.

The project aims are twofold. The first aim is the crafting of a comprehensive virtual reconstruction [19] of the temple complex supported by an evidence-based library of 3D models, sounds, textures and environmental effects (see Figure 2 Top). The second aim is an immersive analytic study of the complex, namely the interactive simulation and tracking of thousands of animated 'agents' as they enter, exit and circulate around the complex (see Figure 2 Bottom).

As an academic exercise that uses visualization and simulation to test how assumptions can be made more precise, the primary users of this system are archaeologists and historians. However, if it could be mediated in an educational context [20] an immersive, virtual exploration of daily life in medieval Angkor would undoubtedly make for an engaging topic in the Australian high school history curriculum, [21] where the civilization of Angkor was recently nominated as a key depth study.
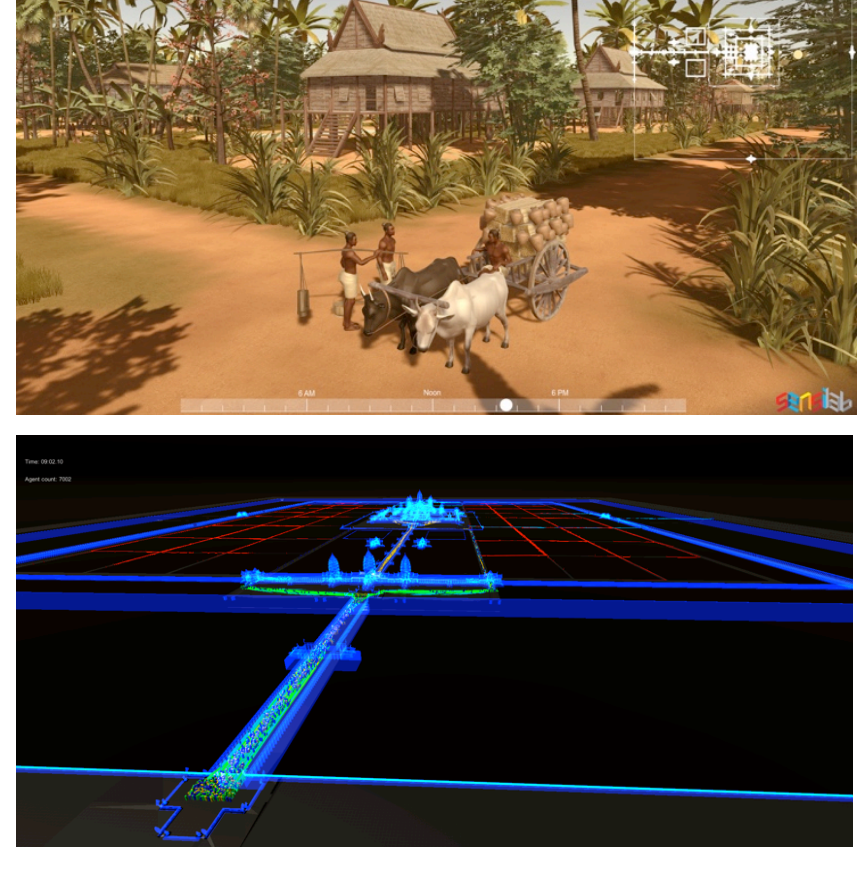

Figure 2. Angkor Wat:

Top: $A$ view of the visualization at ground level, showing animated agents meeting at a crossroad. Interactive overlays include a 24-hour time slider marking the time of day and a superimposed top down map showing the geographical position of the camera. Image by Mike Yeates;

Bottom: A simulation screenshot showing thousands of agents moving in and out of the Angkor Wat complex over several hours. Agent categories

(residents, commuters, suppliers and visitors) are each differentiated by their own color; their activity patterns over time are recorded by persistent motion trails that visualize different scenarios as a 'heat map' Image created by Kingsley Stephens.
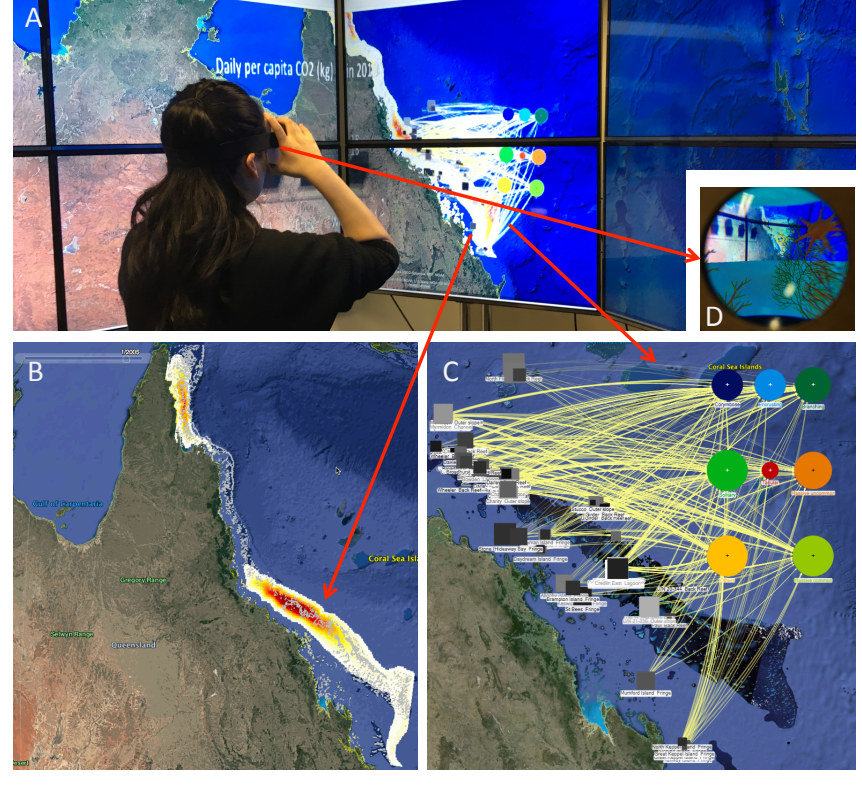

Figure 3. Visualizing Great Barrier Reef: Using immersive analytics to assist education, scientific collaboration, and decision-making to protect the Great Barrier Reef. (A) Real-world snapshots of the implementation of the mix reality system with three integrative components: $(B)$ COTS outbreak density heat map, (C) Reef site versus coral growth forms bipartite graph, and (D) $3 D$ model of corals and COTS in the Google Cardboard (worn by the person in A). 


\section{Visualizing Great Barrier Reef Environmental Effects with Tiled-Display and Augmented Reality}

Recent advances in immersive analytics enable many application domains to accelerate knowledge discovery [1]. The Great Barrier Reef (GBR) in Australia, is one of the world's most important marine eco-systems and a UNESCO World Heritage Site. The GBR has been under constant severe threat over several decades [22], [23] but efforts into improving outreach and public education have only begun relatively recently [24]. At Monash University, we are using a high-resolution tiled display called PerceptuWall (Figure 3 A), with which we investigate how information-rich integrative visualization can help explore comprehensive data sets collected by marine biologists. Example datasets include outbreaks of the Crown of Thorns Seastar (COTS) obtained from the Australian Institute of Marine Science (AIMS) Long-Term Monitoring Program (Figure 3 B), and the 2002 GBR heat stress event that was assessed through multiple AIMS diving expeditions (Figure $3 \mathrm{C}$ ). By integrating and analyzing data from multiple resources, we can generate interactive and immersive visualizations using mixed reality (Figure $3 \mathrm{D}$ ) that are easily expandable to incorporate new datasets from collaborating researchers [25]. The interactive visualization toolkit not only provides analysis and exploration capabilities for researchers, but also makes such data accessible to both expert and non-expert users for the purposes of reef ecology education [25], scientific investigation and decision-making [24].

\section{Stereoscopic Space Map}

The initial idea for the Space Map project was the fact that presentations in CAVEs and CAVE2s are usually optimized for the presenter who is using a wand-like device and head-tracked glassed. In this way, the perspective is optimized for a single person which is not very reasonable for large display environments as their primary strength lays in the fact that they can be used for group presentations.

Therefore, a S3D device was required, which is a) compatible to CAVE2's display configuration, b) provides 3D interaction, and c) head-tracking for simplified navigation. Figure 4 is an anaglyphstereoscopic image which illustrates that the monitor as well as the CAVE2 support passive, polarized glasses.

The Space Map is a World In Miniature (WIM) approach [26], [27]: in our application case it represents the overview of a virtual cell environment using the zSpace. In the Space Map, different tour points can be activated, and the view of CAVE2 is fluently navigating to this specific position. Figure 4 shows the CAVE2 in the background and the zSpace in the foreground. At Figure 4 Bottom a specific tour point was activated and the view navigated showing a specific part of a plant cell: the chloroplast.

Another important feature is the fact that the tour points can also be used to optimize the stereoscopic experience by using our previously introduced interactive projection plane method [28]. In this way, negative aspects, such as ghosting caused by crosstalk, can be minimized [29].

The Space Map software consists of a Java-based extension of the CELLmicrocosmos 4 PathwayIntegration [30] running on the zSpace system, as well as an Omegalib environment running on CAVE2, communicating via Python scripts.

\section{Collaborative Graph Visualization with HMDs \& CAVE2}

As discussed previously, high-quality HMDs are becoming mainstream. These devices potentially represent an affordable alternative to the more traditional, centralized CAVE-style immersive environments, who are mainly set up in universities.
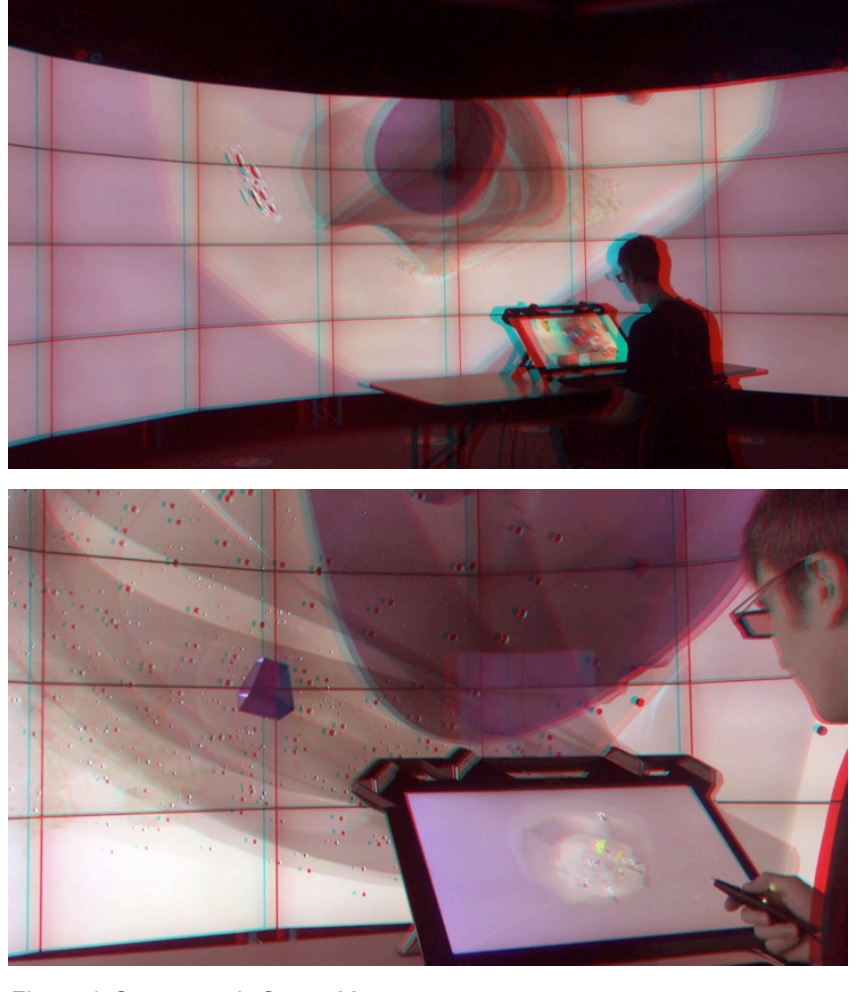

Figure 4. Stereoscopic Space Map.

Top: the ZSpace $200 \otimes$ showing the map of the cell, in the background: the CAVE2 ${ }^{T M}$ environment, showing the nuclear region of the cell;

Bottom: Visiting with the Space Map tour points representing 5 distance classes (red/cyan anaglyph stereo image)
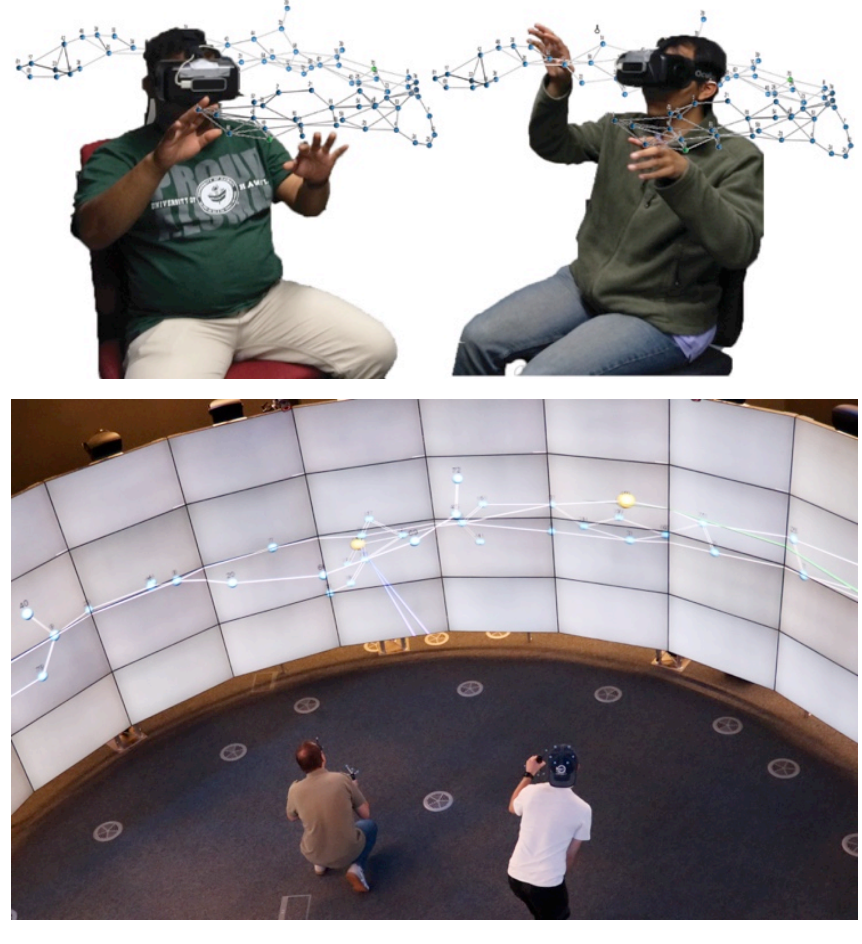

Figure 5. Collaborative Graph Visualization.

Top: Graph Visualization with HMD using the hands for interaction; Bottom: Graph Visualization with CAVE2. 
CAVE-style environments were developed in the context of collaborative sense-making. However, few research has focused on the effectiveness of collaborative visualization in CAVE-style facilities, especially in the context of abstract data visualization tasks, such as networks.

We recently studied how teams of two users collaboratively analyze abstract network visualizations in the CAVE2, and using a pair of connected HMDs (Figure 5) [31]. In the CAVE2 condition, participants were able to walk in the CAVE2 room and see eachother. In the HMDs condition, participants were wearing an Oculus Rift DK2 each, connected on a local network. Participants' head position and finger position were shared on the network. The teams of participants were asked to perform network analysis tasks such as path finding and counting cliques. We established a thorough evaluation frame to identify the differences between the two platforms.

Our results exhibited significant differences between the two conditions in task completion time and the physical movements of the participants within the space: participants using the HMD were faster while the CAVE2 condition introduced an asymmetry in movement between collaborators. Otherwise, affordances for collaborative data analysis offered by the low-cost HMD condition were not found to be different for accuracy and communication with the CAVE2. Our usability questionnaire showed that for this specific task the two platforms were very comparable. However, participants suggested points of improvement for using network HMDs that includes providing a better feedback for face-to-face communication. These results are notable, given that the latest HMDs will soon be accessible (in terms of cost and potentially ubiquity) to a massive audience. We followed up this study by establishing a series of prototypes, concepts and guide lines for remote collaborative visualization in the context of Air Traffic Control and Management data [32].

\section{Swamp City: Visualizing Climate Change Scenarios in Elwood, 2066}

Achieving livable, sustainable and resilient cities of the future requires an in-depth understanding of the complexities, uncertainties and priorities that characterize urban communities, environments and infrastructures. In this context, infrastructure planners, designers and managers are seeking innovative processes that communicate across communities and lead to robust planning outcomes.

This project has developed immersive $3 \mathrm{D}$ visualizations of flooding scenarios to support industry and community stakeholders in understanding the impacts of future urban challenges and the benefits of water sensitive city transformations. It builds upon the existing Cooperative Research Centre for Water Sensitive Cities case study of Elwood (a seaside suburb in Melbourne, Australia), which focused on developing adaptation measures to increase flood resilience and envision a water sensitive future for the area [33].

The 3D simulation being developed allows users to visualize contrasting future scenarios and query aspects of minor and major flood conditions. These interactive scenarios are built upon a parametrically modelled 3D urban map where the simulations envision the Elwood area in its current form and also with proposed adaptations to increase flood resilience.

As well as being showcased at public exhibitions, conferences, seminars and Monash's CAVE 2 environment, these visualizations will be published as online interactives accessible to the broader community (Figure 6).

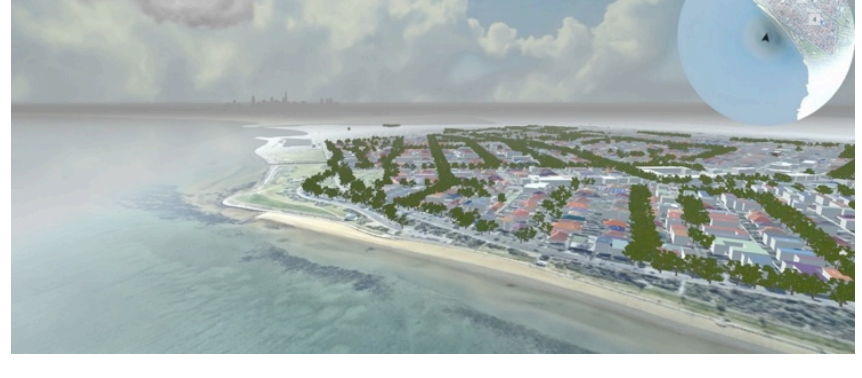

Figure 6. An aerial view in the simulation of the Melbourne suburb of Elwood, as approached from Port Phillip Bay.

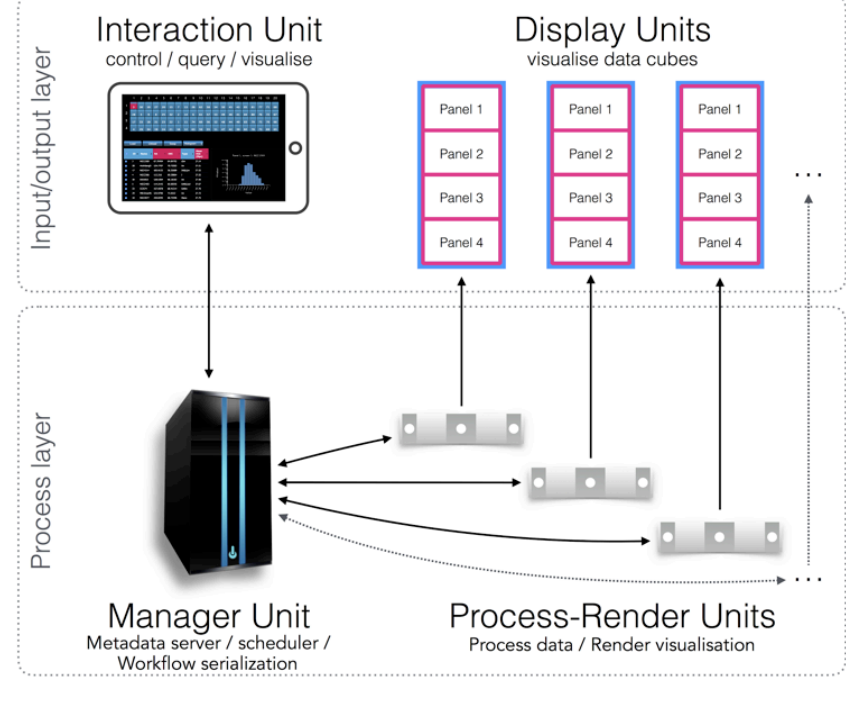

Figure 7. Schematic diagram of encube's framework showing the four types of units and their respective functionalities: An Interaction Unit, A Manager Unit, multiple Process-Render Units, and multiple Display Units.

\section{encube: Large-scale visual analytics framework of volumetric data}

Volumetric images or data cubes are increasingly present in scientific research. Medical imaging routinely generates data cubes using instruments like Positron Emission Tomography (PET), Computerized Tomography (CT) and Magnetic Resonance Imaging (MRI). Ecology, oceanography and Earth sciences gather data cubes - a data structure made of two spatial and one spectral dimensions - using hyperspectral remote sensing from airborne and satellite systems. Astronomers collect data cubes using instruments and facilities like integral field spectrographs and radio interferometers.

Research ventures like the IMAGE-HD study [34], a neuroscience study of Huntington's disease, or the WALLABY Survey [35], a radio astronomy study of the neutral hydrogen content in nearby galaxies, comprise an increasing number of individual observations stored as sets of multidimensional data. In large survey projects, researchers usually face limitations with: 1) interactive visual analytics of sufficiently large subsets of data; 2) synchronous and asynchronous collaboration; and 3) documentation of the discovery workflow.

To better cope with these limitations, we developed encube [36] - a visual analytics framework, with application to high- 
resolution, immersive $3 \mathrm{D}$ environments and desktop displays. encube extends previous comparative visualization systems with:

- the integration of comparative visualization and analysis into a unified framework;

- the documentation of the discovery process; and

- an approach that enables scientists to continue the research process once out of the advanced visualization environment and back at their desktop.

encube is composed of four primary units (Figure 7) providing compute-intensive processing, advanced visualization, dynamic interaction, parallel data query, along with data management. Tablets, smartphones or laptops can be used as interaction units for manipulating, organizing, and querying data within the advanced visualization environment. encube is a modular framework allowing additional functionalities to be integrated as required. Additionally, encube's approach supports a high level of collaboration within the physical environment. encube builds a bridge between high-end display systems (such as CAVE2) and the classical desktop, preserving all traces of the work completed on either platform - allowing to continue the research process wherever you are [37].

When used within an environment like the CAVE2, encube offers a number of advantages over the traditional desktop-based visualization and analysis methodology where a single data cube is examined at a time. Key concepts of encube are what we call 'Single Instruction, Multiple Views,' and 'Single Instruction, Multiple Queries', similar to the concept of 'Single Instruction, Multiple Data' (SIMD). These concepts relate to distributed models of processing and rendering, where one requested action can be applied to many data cubes in parallel. It enables the ability to compare, parameterize or query of order 100 data cubes. Hence, instead of repeating an analysis or a visualization task again and again for many data cubes, the design of encube has the ability to spawn this task to multiple data cubes seamlessly.

Volume rendering is implemented as a custom 3D visualization application based on S2PLOT, and written in C, OpenGL and GLSL [38]. Each Process-Render Unit runs an instance of this application. One of the motivation to using S2PLOT, an open source three-dimensional plotting library, is linked to its 3D stereoscopic rendering capabilities "out of the box". Additionally, S2PLOT comprises multiple customizable methods to handle OpenGL callbacks for interaction, its support of remote input and output via a built-in socket [39]. Moreover, S2PLOT can share its basic rendering transformations (camera position, etc.) with other instances. Additionally, multi-head S2PLOT - a version of S2PLOT distributed over multiple Process-Render Units - or a binding with Omegalib can distribute the rendered visualization over multiple displays, along with tracking capabilities [9].

\section{Stereoscopic Membrane Modeling and Visualization}

Visualization is a crucial part in the analysis pipeline for molecular structures [40], and stereoscopic visualization is a very important aspect supporting the structural analysis of their specific features [28]. Structural biologists were using stereoscopic technologies a decade before the commercial success of $3 \mathrm{D}$ technology around the year 2010.

As currently it is still difficult to observe living biological membranes in nanoscale with microscopes, the simulation of membrane structures plays a key role in biophysics, biochemistry and medical sciences. One of the most popular tools in the biochemical community is Gromacs [41].

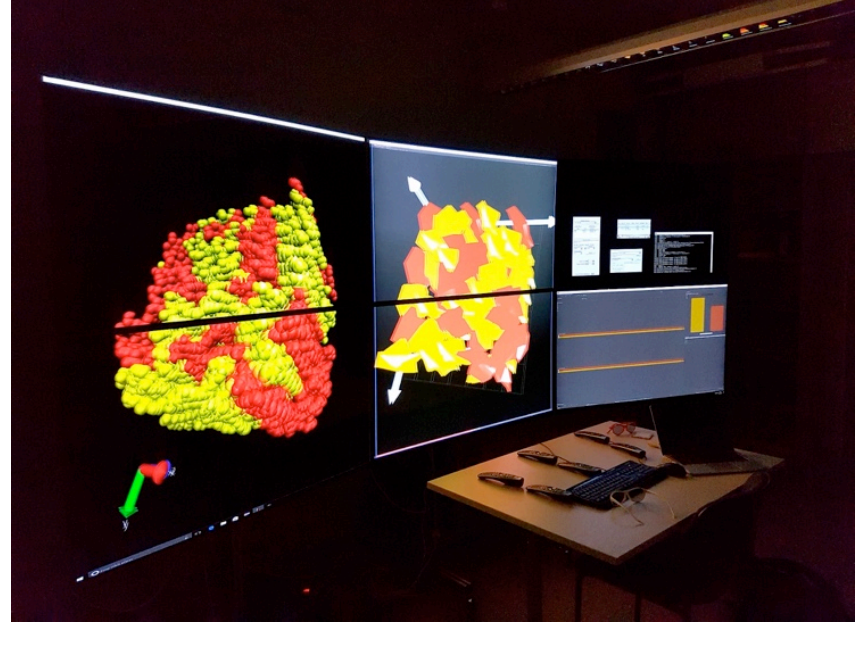

Figure 8. Stereoscopic Membrane Modeling and Visualization: Center TV Column (S3D): Creation of a membrane model by using the CELLmicrocosmos 2 MembraneEditor;

Right TV Column (2D): GUI of the MembraneEditor and VMD;

Left TV Column (S3D): MD Simulation with Gromacs visualized with VMD

There is a growing number of cases where Immersive Analytics-based approaches provided possible solutions for open biomedical problems, e.g. [42]. Because realistic biological membranes are very heterogeneous structures, special tools are required enabling the generation of these highly-specific structures. The CELLmicrocosmos 2 MembraneEditor (CmME) is such a tool which is also supporting S3D visualization [43].

As molecular structures are very complex - partly consisting of millions of atoms - stereoscopic vision is especially important when examining local features of the lipid neighborhood [28]. Figure 8 Center shows the CmME with the initially-generated structure using Lipid Packing Algorithms in S3D. Here, the molecular structures are shown as shapes. After the generation of these structures, we usually simulate them with Gromacs to examine the membrane's behavior over time. Tools like VMD are used to visualize and analyze the trajectories generated with Gromacs [44]. The simulated membrane is shown in VMD in Figure 8 Left, where the spheres represent the Van Der Waals radii of the atoms in S3D. The monitors on the right are used as 2D monitors showing only the GUI elements of both tools.

Whereas CELLmicrocosmos tools support since many years professional S3D compatible to, e.g., NVIDIA Quadro ${ }^{\mathrm{TM}}$ graphics, recently also support for $3 \mathrm{D}$ TVs was implemented by providing side-by-side perspectives. Figure 8 shows the miniCAVE in Konstanz, a Powerwall providing stereoscopic as well as monoscopic visualization by using six $\mathrm{LG}^{\mathrm{TM}}$ Premium $4 \mathrm{~K}$ OLED TVs in a $2 \times 3$ configuration. VMD as well as CmME use side-byside mode here to provide S3D.

In collaboration with Monash Biomedicine Discovery Institute we use at Konstanz University the discussed pipeline to examine the behavior of biological membranes in combination with lipodomics, combining wet lab with in silico research. In this way, we are examining, e.g., the antibiotics resistance of bacterial species, such as Pseudomonas aeruginosa or Escherichia coli. Figure 8 shows the composition of such a model membrane which was simulated based on experimental data. The $2 \mathrm{D}$ view on the right side of the figure shows GUI elements of VMD and CmME, which is currently showing the lipid percentage distribution as bar charts. 


\section{Conclusions and Outlook}

Here, we presented a number of Immersive Analytics-related projects developed by three visualization facilities. Seven projects were introduced. Visualization-driven historical research is conducted by the remodeling and crowd simulation of the medieval metropole Angkor Wat. Environmental changes were visualized and analyzed in Elwood, a flood-threatened suburb at Melbourne's seaside, as well as in the ocean for the Great Barrier Reef by combining a Powerwall with mixed-reality techniques. A Powerwall was also used for the visual analysis of the modeling and simulation of membranes in the context of biomedical research. On the more technical side, collaborative graph visualization was evaluated by comparing HMDs with the CAVE2. Then, the Stereoscopic Space Map, a new navigation interface for the CAVE2 was implemented by connecting it to a Fishtank-based technology, the zSpace. And finally, a new visual analytics framework for the CAVE2 was introduced providing advanced volume rendering capabilities: encube.

For all of these projects the integration of S3D technology plays a crucial role and we also shortly discussed the applied technologies: CAVEs, Powerwalls, Fishtanks, and HMDs.

In the future we are planning to implement S3D support also for those approaches which currently support only standard 3D. For example, we are thinking about integrating S3D functionality into our web-based approaches, such as CereVA or CmPIweb [45], [46].

Also, there is another recently-published project which is especially interesting for collaborations between different distant research facilities like in case of Monash University and the University of Konstanz: ContextuWall. Here, we would like to introduce this eights project which is also planning to integrate stereoscopic functionality in the near future.

\section{ContextuWall}

ContextuWall [47] is a system to support local and remote collaboration using large display systems making use of professional and consumer grade hardware. It enables users to easily share content such as images using various touch enabled interaction clients. This content can then be graphically annotated using the ContextuWall client software. To enable remote collaboration multiple ContextuWall systems can be connected over the internet to create one virtual desktop to display and arrange the same content in real time on multiple sites supporting multiple display configurations and resolutions driven by the appbased SAGE2 [48] framework (Figure 9).

To overcome the current limitation of sharing and annotating 2D content only, the ContextuWall system will be extended towards supporting static and dynamic 3D content using standard as well as stereoscopic display technologies. Hence the SAGE2 framework will be extended to incorporate mixed 2D and projected/stereoscopic 3D content in a browser window. Besides overlaying annotations on top of shared 2D content, annotation of 3D content will be possible in two modes: 1) placement of drawn $2 \mathrm{D}$ annotations as textures in 3D space, and 2) integration of brushing annotations in 3D space. The first mode requires the extension of the interaction client to enable annotation of 3D content by providing a $2 \mathrm{D}$ annotation canvas which can then be placed as texture in 3D space. The second mode will make use of 3D tracked wand hardware to enable brushing annotation of the content displayed.

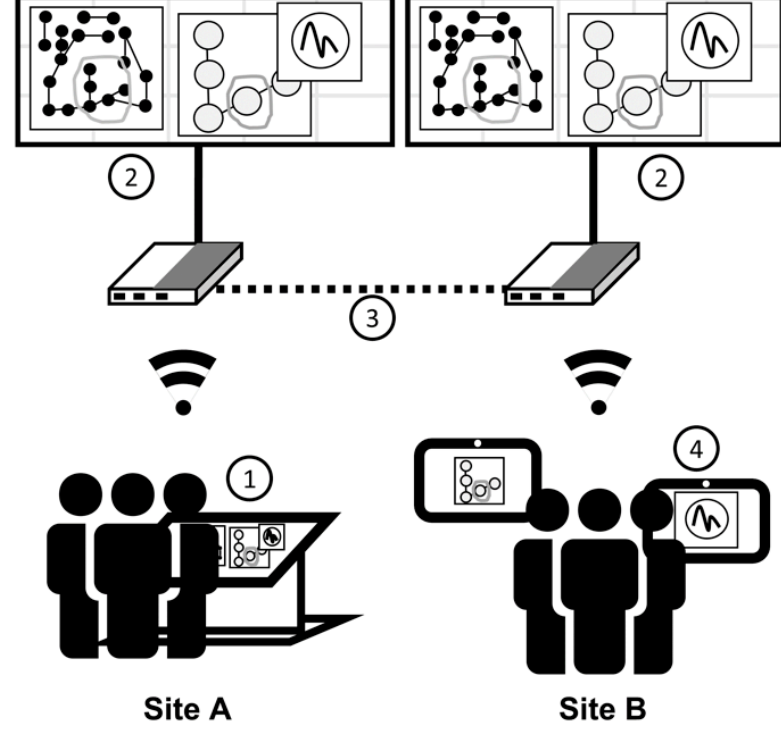

Figure 9. Application of the ContextuWall system where site $A$ uses a ContextuWall-enabled software to share $2 D$ and $3 D$ content (1) on their display wall (2) as well as sending them to the remote site over a secure connection (3). Both sites can simultaneously annotate content using the touch enabled interaction clients (1),(4).

\section{Abbreviations}

$\begin{array}{lll}\text { - } & 2 D & \text { two-dimensional } \\ \text { - } & \text { AIMS } & \text { three-dimensional } \\ \text { - } & \text { CAVE } & \text { CAVE Autralian Institute of Marine Science } \\ \text { - } & \text { CmCX } & \text { CELLmicrocosmos 1.2 CellExplorer } \\ \text { - } & \text { CmME } & \text { CELLmicrocosmos 2.2 MembraneEditor } \\ \text { - } & \text { CmPI } & \text { CELLmicrocosmos 4.2 PathwayIntegration } \\ \text { - } & \text { COTS } & \text { Crown of Thorns Seastar } \\ \text { - } & \text { CT } & \text { Computerized Tomography } \\ \text { - } & \text { GBR } & \text { Great Barrier Reef } \\ \text { - } & \text { HCI } & \text { Human Computer Interaction } \\ \text { - } & \text { HMD } & \text { Head-Mounted Display } \\ \text { - } & \text { MD } & \text { Molecular Dynamics } \\ \text { - } & \text { MIVP } & \text { Monash Immersive Visualization Platform } \\ \text { - } & \text { MRI } & \text { Magnetic Resonance Imaging } \\ \text { - } & \text { PET } & \text { Positron Emission Tomography } \\ \text { - } & \text { S3D } & \text { Stereoscopic 3-dimensional (visualization) } \\ \text { - } & \text { SIMD } & \text { Single Instruction, Multiple Data } \\ \text { - } & \text { VMD } & \text { Visual Molecular Dynamics } \\ \text { - } & \text { WIM } & \text { World In Miniature }\end{array}$

\section{Acknowledgement}

H.N. would like to thank Stephen Wang and his team for the development of the mixed reality visualisation prototype. Work is partially supported by the Richard Pratt Fellowship in Prostate Cancer and ARC Discovery Grant DP130104792 to SEB. The Australian Regenerative Medicine Institute is supported by grants from the State Government of Victoria and the Australian Government.

B.S. and Y.Z. would like to thank Monash University for receiving the Microbiology@Monash Collaborative Grant in 2016.

$B . S$. and K.K. would like to thank Mr. Wind and Mr. Moser for helping us to assemble the miniCAVE in Konstanz (Figure 8) one day before the submission deadline, so just in time! 


\section{References}

[1] T. Chandler et al., "Immersive Analytics," in Big Data Visual Analytics (BDVA), 2015, pp. 1-8.

[2] D. A. Keim, F. Mansmann, J. Schneidewind, J. Thomas, and H. Ziegler, "Visual analytics: Scope and challenges," in Visual data mining, Springer, 2008, pp. 76-90.

[3] K. A. Cook and J. J. Thomas, "Illuminating the path: The research and development agenda for visual analytics," Pacific Northwest National Laboratory (PNNL), Richland, WA (US), 2005.

[4] N. Greffard, F. Picarougne, and P. Kuntz, "Visual community detection: An evaluation of 2D, 3D perspective and 3D stereoscopic displays," in International Symposium on Graph Drawing, 2011, pp. 215-225.

[5] C. Cruz-Neira, D. J. Sandin, T. A. DeFanti, R. V. Kenyon, and J. C. Hart, "The CAVE: audio visual experience automatic virtual environment," Commun. ACM, vol. 35, no. 6, pp. 64-72, 1992.

[6] A. Febretti et al., "CAVE2: a hybrid reality environment for immersive simulation and information analysis," in IS\&T/SPIE Electronic Imaging, 2013, pp. 864903-864903-12.

[7] W. J. Goscinski et al., "The multi-modal Australian ScienceS Imaging and Visualization Environment (MASSIVE) high performance computing infrastructure: applications in neuroscience and neuroinformatics research," Front. Neuroinformatics, vol. 8, 2014.

[8] “CAVE2 | Mechdyne." [Online]. Available: https://www.mechdyne.com/hardware.aspx?name=CAVE2. [Accessed: 05-Jan-2017].

[9] A. Febretti, A. Nishimoto, V. Mateevitsi, L. Renambot, A. Johnson, and J. Leigh, "Omegalib: A multi-view application framework for hybrid reality display environments," in Virtual Reality (VR), 2014, pp. 9-14.

[10] “StereoMirror 3D Documentation,” Planar. [Online]. Available: /support/products/3d-stereoscopic/. [Accessed: 05-Jan-2017].

[11] "moreSonic ${ }^{3} 2720 \mathrm{H}$ - Beam Splitter 3D Stereo Display with Sonic Stereo Mirror - more3D.” [Online]. Available: http://www.more3d.com/more3d/index.php?id=236. [Accessed: 05Jan-2017].

[12] more3D, "more3D - Technology Comparison Workstation 3D Stereo Displays 2015." [Online]. Available: http://www.more3d.com/more3d/files/Downloads/Technology\%20 Comparison\%20Workstation\%203D\%20Stereo\%20Displays\%202 015.pdf. [Accessed: 05-Jan-2017].

[13] “zSpace Product,” 2015. [Online]. Available: http://zspace.com/product. [Accessed: 02-Jun-2015].

[14] I. E. Sutherland, "A head-mounted three dimensional display," in Proceedings of the December 9-11, 1968, fall joint computer conference, part I, 1968, pp. 757-764.

[15] C. J. Fluke and D. G. Barnes, "The Ultimate Display," in Proceedings of Astronomical Data Analysis Software and Systems (ADASS) XXV, Sydney, in press, 2016.

[16] D. H. Evans et al., "Uncovering archaeological landscapes at Angkor using lidar," Proc. Natl. Acad. Sci., vol. 110, no. 31, pp. 12595-12600, 2013.
[17] M. T. Stark, D. Evans, C. Rachna, H. Piphal, and A. Carter, "Residential patterning at Angkor Wat," Antiquity, vol. 89, no. 348, pp. 1439-1455, 2015.

[18] M. Pfeiffer, C. Carré, V. Delfosse, P. Hallot, and R. Billen, "Virtual Leodium: From an historical 3D city scale model to an archaeological information system," in International Committee for Documentation of Cultural Heritage (CIPA). Symposium: Recording, Documentation and Cooperation for Cultural Heritage, 2013.

[19] T. Chandler and M. Polkinghorne, "Through the visualisation lens: Temple models and simulated context in a virtual Angkor," in Old Myths and New Approaches: Interpreting Ancient Religious Sites in Southeast Asia, Monash University Publishing, 2012, pp. 218-236.

[20] T. Chandler, "Playing Angkor: Exploring the historical and archaeological themes of the Khmer empire through game engine technologies," in Across Space and Time: Papers from the 41st Conference on Computer Applications and Quantitative Methods in Archaeology, Perth: Amsterdam University Press, 2015, pp. 379385.

[21] N. Cummins and T. Chandler, "Teaching Angkor and the Khmer Empire with 3D technologies," Agora, vol. 49, no. 4, p. 59, 2014

[22] G. De'ath, K. E. Fabricius, H. Sweatman, and M. Puotinen, "The 27-year decline of coral cover on the Great Barrier Reef and its causes," Proc. Natl. Acad. Sci., vol. 109, no. 44, pp. 17995-17999, 2012.

[23] A. S. Wenger et al., "Effects of reduced water quality on coral reefs in and out of no-take marine reserves," Conserv. Biol., vol. 30, no. 1, pp. 142-153, 2016.

[24] H. Nim, T. Done, F. Schreiber, and S. Boyd, "Interactive geolocational and coral compositional visualisation of Great Barrier Reef heat stress data," in Big Data Visual Analytics (BDVA), 2015, pp. $1-7$.

[25] H. T. Nim et al., "Communicating the Effect of Human Behaviour on the Great Barrier Reef via Mixed Reality Visualisation," in Big Data Visual Analytics (BDVA), 2016, pp. 1-6.

[26] R. Stoakley, M. J. Conway, and R. Pausch, "Virtual reality on a WIM: interactive worlds in miniature," in Proceedings of the SIGCHI conference on Human factors in computing systems, 1995, pp. 265-272.

[27] B. Sommer et al., "Stereoscopic Space Map - Semi-immersive Configuration of 3D-stereoscopic Tours in Multi-display Environments," Electron. Imaging Proc. Stereosc. Disp. Appl. XXVII, vol. 2016, no. 5, pp. 1-9, Feb. 2016.

[28] B. Sommer, C. Bender, T. Hoppe, C. Gamroth, and L. Jelonek, "Stereoscopic cell visualization: from mesoscopic to molecular scale," J. Electron. Imaging, vol. 23, no. 1, pp. 011007-1-011007$10,2014$.

[29] A. J. Woods, "How are crosstalk and ghosting defined in the stereoscopic literature?," in IS\&T/SPIE Electronic Imaging, 2011, p. 78630Z-78630Z-12.

[30] B. Sommer, J. Künsemöller, N. Sand, A. Husemann, M. Rumming, and B. Kormeier, "CELLmicrocosmos 4.1: an interactive approach to integrating spatially localized metabolic networks into a virtual 3D cell environment," in Proceedings of BIOSTEC 2010, 2010, pp. 90-95. 
[31] M. Cordeil, T. Dwyer, and C. Hurter, "Immersive solutions for future Air Traffic Control and Management," in Proceedings of the 2016 ACM Companion on Interactive Surfaces and Spaces, 2016, pp. 25-31.

[32] M. Cordeil, T. Dwyer, K. Klein, B. Laha, K. Marriott, and B. H. Thomas, "Immersive Collaborative Analysis of Network Connectivity: CAVE-style or Head-Mounted Display?," IEEE Trans. Vis. Comput. Graph., vol. 23, no. 1, pp. 441-450, 2017.

[33] B. C. Rogers and A. W. Gunn, "Towards a Water Sensitive Elwood: A Community Vision and Transition Pathways - Mapping Water Sensitive City Scenarios (Project A4.2)," Monash University, Melbourne, Technical/Milestone Report A4.2-2-2015, 2015.

[34] N. Georgiou-Karistianis et al., "Automated differentiation of prediagnosis Huntington's disease from healthy control individuals based on quadratic discriminant analysis of the basal ganglia: the IMAGE-HD study," Neurobiol. Dis., vol. 51, pp. 82-92, 2013.

[35] B. S. Koribalski and L. Staveley-Smith, ASKAP Survey Science Proposal. 2009.

[36] D. Vohl et al., "Large-scale comparative visualisation of sets of multidimensional data," PeerJ Comput. Sci., vol. 2, p. e88, 2016.

[37] D. Vohl, C. J. Fluke, A. H. Hassan, D. G. Barnes, and V. A. Kilborn, "Collaborative visual analytics of radio surveys in the Big Data era," ArXiv Prepr. ArXiv161200920, 2016.

[38] D. G. Barnes, C. J. Fluke, P. D. Bourke, and O. T. Parry, “An advanced, three-dimensional plotting library for astronomy," Publ. Astron. Soc. Aust., vol. 23, no. 2, pp. 82-93, 2006.

[39] "OpenGL - The Industry Standard for High Performance Graphics." [Online]. Available: https://www.opengl.org/. [Accessed: 05-Jan-2017].

[40] J. D. Hirst, D. R. Glowacki, and M. Baaden, "Molecular simulations and visualization: introduction and overview," Faraday Discuss., vol. 169, pp. 9-22, 2014.

[41] B. Hess, C. Kutzner, D. van der Spoel, and E. Lindahl, "Gromacs 4: Algorithms for highly efficient, load-balanced, and scalable molecular simulation," J Chem Theory Comput, vol. 4, no. 3, pp. 435-447, 2008.

[42] M. Dreher et al., "Interactive molecular dynamics: scaling up to large systems," Procedia Comput. Sci., vol. 18, pp. 20-29, 2013.

[43] B. Sommer et al., "CELLmicrocosmos 2.2 MembraneEditor: a modular interactive shape-based software approach to solve heterogeneous Membrane Packing Problems," J. Chem. Inf. Model., vol. 5, no. 51, pp. 1165-1182, 2011.

[44] W. Humphrey, A. Dalke, and K. Schulten, "VMD: Visual Molecular Dynamics," J. Mol. Graph., vol. 14, no. 1, pp. 33-38, 1996.

[45] G. Kovanci, M. Ghaffar, and B. Sommer, "Web-based hybriddimensional Visualization and Exploration of Cytological Localization Scenarios," J. Integr. Bioinforma., vol. 13, no. 4, p. 298, 2016.

[46] M. de Ridder, K. Klein, and J. Kim, "CereVA - Visual Analysis of Functional Brain Connectivity," in Proceedings of the 6th International Conference on Information Visualization Theory and Applications (VISIGRAPP 2015), 2015, pp. 131-138.
[47] M. Klapperstuck et al., "ContextuWall: Peer Collaboration Using (Large) Displays," in Big Data Visual Analytics (BDVA), 2016, pp. $1-8$.

[48] L. Renambot et al., "SAGE2: A collaboration portal for scalable resolution displays," Future Gener. Comput. Syst., vol. 54, pp. 296-305, 2016. 